\title{
Image registration demonstrates the growth plate has a variable affect on vertebral strain
}

\author{
M. R. Hardisty, M. Akens, A. J. Yee, and C. M. Whyne \\ Version Post-Print/Accepted Manuscript \\ Citation Hardisty MR, Akens M, Yee AJ, Whyne CM. Image registration \\ (published version) demonstrates the growth plate has a variable affect on vertebral strain. \\ Ann Biomed Eng. 2010 Sep;38(9):2948-55. doi: 10.1007/s10439-010- \\ 0052-0. PMID: 20443059 \\ Publisher's Statement The final published version of this article is available at Springer Verlag \\ via https://dx.doi.org/10.1007/s10439-010-0052-0.
}

Always cite the published version, so the author(s) will receive recognition through services that track citation counts, e.g. Scopus. If you need to cite the page number of the TSpace version (original manuscript or accepted manuscript) because you cannot access the published version, then cite the TSpace version in addition to the published version using the permanent URI (handle) found on the record page. 


\title{
Image Registration Demonstrates a Variable Effect of the Growth Plate on Vertebral Strain
}

\author{
M. R. Hardisty, M. Akens, A. Yee, and C. M. Whyne \\ Orthopaedic Biomechanics Laboratory, Sunnybrook Health Sciences Centre, University of Toronto, 2075 Bayview Ave., \\ Room UB-19, Toronto, ON M4N 3M5, Canada
}

(Received 4 December 2009; accepted 16 April 2010)

Associate Editor Peter E. McHugh oversaw the review of this article.

\begin{abstract}
Characterizing the biomechanical behavior of the vertebrae is important in understanding the impact of structural and material changes on spinal growth and fracture risk. The growth plate is critical for the normal development of the skeleton, with abnormalities leading to uneven maturation. Little is known about how growth plates affect the stress and strain experienced by the surrounding bone. Concentrated strain within the growth plate may influence mechanical cell signaling during development, lead to increased fracture risk at this site and may influence average bone strain measures. It is hypothesized that the growth plates and adjacent bony areas will take up a large amount of the strain within rat-tail vertebrae under axial compressive loading, leading to increased average bone strain measures. The sixth caudal vertebrae of $8 \mathrm{rnu} / \mathrm{rnu}$ rats were $\mu \mathrm{CT}$ scanned in both loaded (20-32 $\mathrm{N}$ axial compression) and unloaded configurations. Image registration was used to calculate strain in the bone due to the applied load by finding a spatial mapping between the two scans. In seven of the eight rats, the majority of the strain measured within their vertebrae was concentrated in the growth plates. Five of the specimens had growth plates that demonstrated rigid behavior in contrast to compliant growth plate behavior seen in the other three rats. The presence of a compliant growth plate led to higher average $(-0.03$ vs. -0.01$)$ and maximum $(-0.13$ vs. -0.02 ) strains. The strain within the growth plate is important to consider when interpreting apparent tissue level biomechanical data commonly reported in the literature [Ito, M., et al. Contribution of trabecular and cortical components to the mechanical properties of bone and their regulating parameters. Bone 31:351-358, 2002; Lauing, K., et al. Binge alcohol treatment of adolescent rats followed by alcohol abstinence is associated with site-specific differences in bone loss and incomplete recovery of bone mass and strength. Alcohol 42:649-656, 2008; Sehmisch, S., et al. Short-term effects of parathyroid hormone on rat lumbar vertebrae. Spine (Phila Pa 1976), 34:2014-2021, 2009;
\end{abstract}

Address correspondence to C. M. Whyne, Orthopaedic Biomechanics Laboratory, Sunnybrook Health Sciences Centre, University of Toronto, 2075 Bayview Ave., Room UB-19, Toronto, ON M4N 3M5, Canada. Electronic mail: cari.whyne@sw.ca
Shiraishi, A., et al. The combination therapy with alfacalcidol and risedronate improves the mechanical property in lumbar spine by affecting the material properties in an ovariectomized rat model of osteoporosis. BMC Musculoskelet. Disord. 10:66, 2009], as this study suggests strains are not uniformly distributed with high concentrations in and around the growth plate. This strain distribution may provide insight into the mechanical signals that cells experience during the formation of new bone, with the higher strains near the growth plate signaling cells to lay down more bone, while also leading to increased risk of fracture in this region.

Keywords-Growth plate, Biomechanics, Digital image correlation, Spine, Vertebrae.

\section{INTRODUCTION}

Characterizing the biomechanical behavior of the vertebrae is important in understanding the impact of structural and material changes on spinal growth and fracture risk. Vertebral trabecular bone at the apparent material level has been demonstrated to fail at a strain of 0.008 independent of density ${ }^{15}$; however, the measurements of apparent behavior used to identify this value may not necessarily be indicative of trabecular tissue level strain. Tissue level strains in trabecular bone have been shown to differ from the applied apparent level strains through the use of voxel-based finite element modeling of trabecular bone cores. ${ }^{6,7}$

Human vertebrae contain both an inferior and superior growth plate which normally fuse following puberty. ${ }^{1}$ The growth plate is critical for the normal development of the skeleton, with abnormalities leading to uneven maturation. The rate of bone lengthening is known to be affected by externally applied loading. In the spine, developmental pathologies of the growth plates can result in spondylolisthesis 
and scoliosis. ${ }^{26}$ It would, therefore, be advantages to have a greater understanding of the growth plate's response to loading within a physiologic loading environment.

The growth plate is initially a dynamic structure that contains proliferating chondrocytes that create an extracellular matrix much like cartilaginous tissue and as such has a much lower modulus than bone. ${ }^{29}$ As bone growth occurs, the growth plate tissue becomes mineralized. This rigid behavior may represent more mineralized growth plate tissue, a thinner growth plate, or regions of bony bridging, yielding a more rigid construct.

Rodents are often used as preclinical models for investigating the biomechanical consequences of spinal pathologies and therapeutic interventions. ${ }^{9}$ Growth plates are present within rat vertebrae throughout life ${ }^{13}$ (Fig. 1). Little is known about how growth plates affect the stress and strain experienced by the surrounding bone. As such it is important to consider the impact of growth plates in understanding the biomechanical behavior of whole vertebrae in rodent models.

In contrast to physical strain gage measurements or global changes in displacement measured during biomechanical testing, image registration of $\mu \mathrm{CT}$ scans can be used to spatially resolve full strain fields. Deformable registration techniques have been previously applied to quantify tissue strain. $2,12,17,23,25,27,30$ Patterns of intensity used to spatially align images provide a method of back calculating tissue strain in both bone and soft tissues. This investigation aims to characterize the biomechanical response of rat-tail vertebrae to axial compressive loading through imagebased strain measurement. It is hypothesized that the growth plates and adjacent bony areas will be responsible for a large amount of the strain generated within rat-tail vertebrae under axial compressive loading, leading to increased average bone strain measures.

\section{METHODS}

The sixth caudal vertebrae of $8 \mathrm{rnu} / \mathrm{rnu}$ rats (89 weeks of age) were $\mu \mathrm{CT}$ scanned (GE Explore Locus, General Electric Company, Fairfield, CT, USA) first in an unloaded configuration and subsequently in a loaded configuration $(20-32 \mathrm{~N}$ axial compression to an equivalent stress of $\sim 10 \mathrm{MPa})$. The mechanical testing was designed to maximize the prefailure load to the specimens in order to generate a sufficiently high-strain magnitude. Larger strain magnitudes increase the certainty of the analytic results (increasing the signal-tonoise ratio), lessening the impact of scanner resolution and any motion that may have occurred as a result of creep. Further, in order to maximize the strain applied to the specimens, the amount of prefailure load that each specimen could safely bear was individually estimated. The estimates were based upon the stability of the pins within the adjacent vertebrae; in a few pilot specimens, fracture occurred during load application in the vertebrae through which the load was applied (adjacent to the vertebral body of interest). The pins holes in these vertebrae were always the site of fracture because they created stress risers within the bone that would not otherwise have been present.

Imaging was carried out over 907 views covering $360^{\circ}$ of rotation $(2.5 \mathrm{~h}$ scan time $)$ with an X-ray source at $90 \mu \mathrm{A}$ and $80 \mathrm{kV}$. Reconstruction of volumes from
118

119

120

121

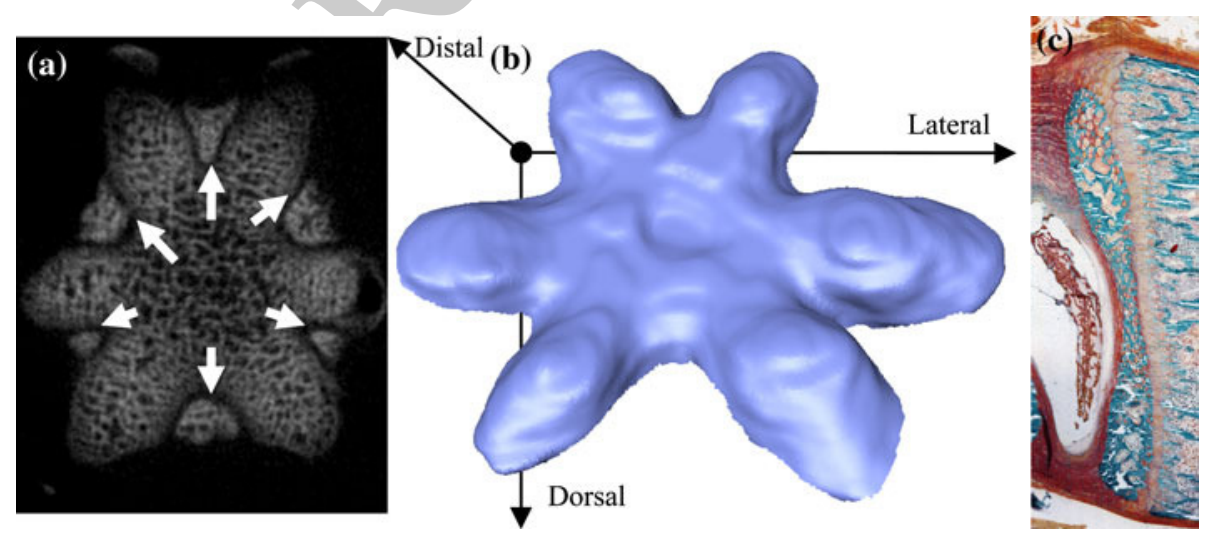

FIGURE 1. (a) MicroCT cross-section of the growth plate within the sixth caudal vertebrae of rnu/rnu rats. The growth plate appears as a dim, less dense line (white arrows) between two relatively much more dense regions of bone. The growth plate in these vertebrae has a characteristic undulated structure that cannot be entirely captured in a single cross-section. (b) A 3D reconstruction of the growth plate from a manual segmentation of the CT data, more clearly showing the undulated structure. (c) A histological nondecalcified section depicting the vertebral growth plate in rnu/rnu rats stained with Goldner-Masson that stains cell nucleuae dark brown to black, cytoplasm and muscle brick red, connective tissue, bone and acidic mucous substances stain green. The growth plate is well defined by the absence of calcified tissue, appearing as a white band within the structure. Left is distal. 
the X-ray projections was carried out with the GE Explore Locus Recon utility to $17.5 \mu \mathrm{m} /$ voxel. The unloaded configuration of the specimen was imaged while immersed in agar. The load was applied to samples with custom designed $\mu \mathrm{CT}$ compatible spring loaded telescoping device (Fig. 2); loads applied to the samples were allowed to creep for $30 \mathrm{~min}$. After $30 \mathrm{~min}$, the viscoelastic response of the tissues loaded was found in calibration experiments to have sufficiently dissipated. The device was designed to transfer axial compressive loads to the vertebra of interest through the adjacent vertebrae and intervertebral discs of the excised spinal motion segment (fifth to seventh caudal vertebrae). Previous pilot work had indicated the potential of this configuration for applying axial compressive loading to vertebrae in intact rat-tails. ${ }^{3}$ The device was calibrated by loading select specimens inline with a ELFM-T2M, $500 \mathrm{~N}$ capacity subminiature tension/compression load cell (Entron Devices Inc., Fairfield, CT, USA) and by calibration against a materials testing machine $250 \mathrm{lbs}$ load cell (MTS Bionix 858, Eden Prairie, MN, USA) in a specialized calibration set up.

A digital volume correlation method, previously demonstrated and validated, relying on image registration was used to calculate strain and displacement fields in the bone due to the applied load. ${ }^{10}$ This technique utilizes both loaded and unloaded images of the specimen. The registration was implanted in a multiresolution framework. The algorithm begins with an iterative optimization of affine mapping parameters to ideally match up the entire loaded and unloaded scans based upon a mutual information metric. The affine mapping parameters allow a transform with rotation, scaling, shearing, and translation (12 DOF). Once an acceptable fit was obtained, the moving scan was partitioned into eight pieces by bisecting the scan along the three axes. The affine transform found for registering the whole moving scan was used as the initial guess in these eight subpieces. These eight pieces formed the second level of registration and were each individually registered. The subpieces then each spawned eight more individual pieces and so on until the maximum level of analysis desired was reached. Once the registration was completed, the displacement field and strain field were calculated:

$$
\begin{aligned}
e & =\frac{1}{2}\left(\nabla \vec{A}^{T}+\nabla \vec{A}\right) \\
\vec{A}(x, y, z) & =\tilde{T}(x, y, z) P(x, y, z)-P(x, y, z) \\
T & =\left[\begin{array}{llll}
J_{11} & J_{12} & J_{13} \mid & X_{\text {Trans }} \\
J_{21} & J_{22} & J_{23} \mid & Y_{\text {Trans }} \\
\frac{J_{31}}{X_{\text {Center }}} & \frac{J_{32}}{Y_{\text {Center }}} & \frac{J_{33}}{Z_{\text {Center }}} & 1
\end{array}\right]
\end{aligned}
$$

where $e$ is the strain matrix, $A$ is the displacement vector, $T$ is the affine transform found from registration, and $P$ is the voxel location. The gradient of the displacement field and strain were calculated at the center of registration regions with finite differencing; elsewhere the fields were interpolated using third order B splines (Insight Segmentation Toolkit, Bethesda, MD, USA).

Regions that were individually registered and used to calculate the strain varied from $260 \times 240 \times$ $170 \mu \mathrm{m}^{3}$ to $750 \times 840 \times 580 \mu \mathrm{m}^{3}$. The $z z$ strain distributions in the rat-tail were characterized by plotting strain contours, calculating the mean strain, median strain and strain in the 10th and 90th percentile of the strain distribution.
185

186

187

188

189

190

191

192

193

194

195

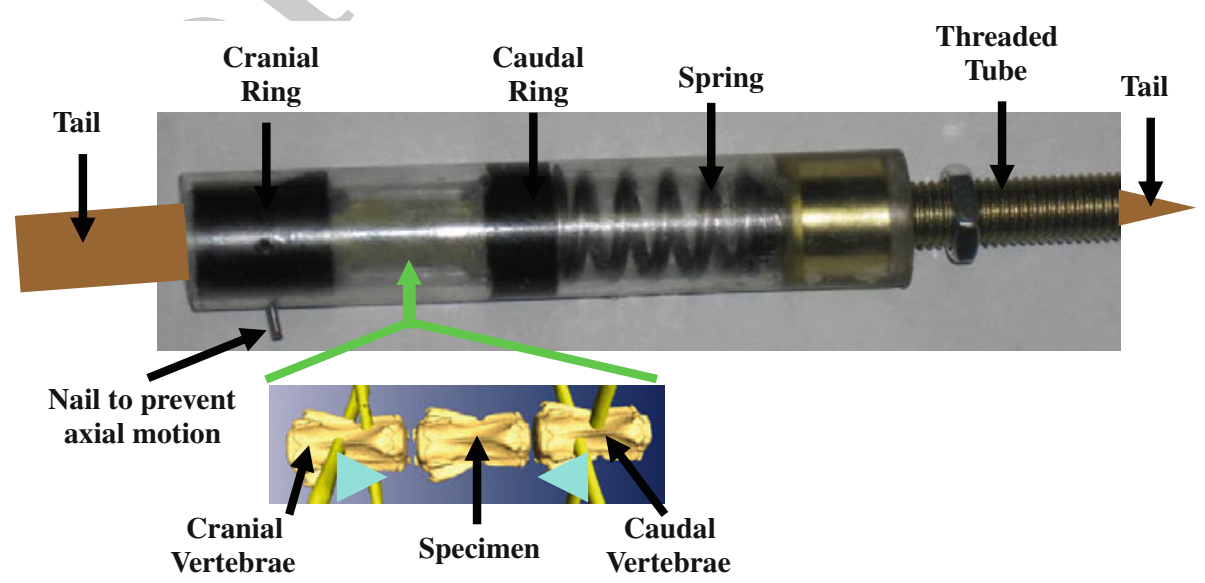

FIGURE 2. Custom designed CT compatible spring loaded telescoping device for application of axial compressive loading to rat-tail spinal motion segments. 
Axial strain was successfully calculated in whole rat vertebrae by image registration, ranging from 0.008 to 0.04 in compression with an average axial strain of 0.016 (Table 1). None of the vertebrae showed evidence of damage due to the applied loading. The nature of the growth plate largely determined the strain magnitude and distribution, as evident in the contour plots of the strain field (Fig. 3). In seven of the eight rats, the majority of the strain measured within their vertebrae was concentrated in the growth plates and surrounding bone.

Interestingly, the structural behavior of the rat-tail growth plates was not consistent across specimens. The specimens yielded two distinct strain patterns based on the rigidity of their growth plates [rigid growth plates $(n=5)$ and compliant growth plates $(n=3)$ ] (Table 2). The presence of a compliant growth plate led to maximum axial strains exceeding 0.1 (0.1-0.16 in compression) compared to maximum axial strains in the more rigid growth plate group remaining below 0.04 (0.022-0.032 in compression) (Fig. 4). The rigid growth plates were thinner and deformed much less, consistent with the strain measurements.

Centrally located strain concentrations of low magnitude and more limited spatial extent were observed in the vertebral trabecular bone (Fig. 3). All rats showed elevated strains in trabecular regions of the bone: four in the more caudal regions, three in the more cranial regions and one evenly distributed between the two regions. In five of the specimens, the strain distributions were consistent with primarily axial compressive loading. The other specimens had strain distributions that indicated loading was slightly asymmetric, causing limited bending and shearing behavior.
MicroCT-based image registration techniques were successful in determining the full strain field present in rat-tail vertebrae under axial compressive loading. The growth plates were found to absorb the majority of the strain within the rat vertebrae; however, the behavior of the growth plates was not consistent across all specimens. The results confirm the hypothesis that the growth plates increase the average strains within the vertebrae. However, the results do not demonstrate a uniform effect. Although 8- to 9-week-old rnu/rnu rats were used in this analysis, the rodent spine may have some inconsistency in its level of skeletal maturity, leading to altered mechanical behavior. Villemure and Stokes have shown that rat tibial growth plate's effective modulus increases with the age of the animal. $^{29}$

The variability of compliance in the growth plate is important to consider when interpreting biomechanical data generated from axial loading of rat vertebrae. The standard protocol for measuring strength of bony specimens of applying an increasing compressive load will give a false picture of the strain during the elastic phase of loading. Increasing loading will first compress the growth plate and only following compression of this structure will significant displacement and strain occur in the trabecular bone and vertebral shell. This observation of how a growth plate structure will effect the strain experienced by the surrounding bone tissue is supported by investigation of growth plate tissue material properties within rodents, ${ }^{28}$ rabbits ${ }^{8,18}$ and bovine specimens, ${ }^{4,5}$ both in tension, ${ }^{4,8,28}$ and compression. ${ }^{5}$ Growth plate material properties have been found to vary in the lateral plane, with the interior ${ }^{5}$ and the anterior ${ }^{4}$ parts of the growth plate found to be the stiffest and strongest. The aggregate modulus of the growth plate has been found to be lower in
250

251

252

253

254

255

256

257

258

259

260

261

262

263

264

265

266

267

268

269

270

271

272

273

274

275

276

277

278

279

280

281

282

283

284

285

TABLE 1. Summary of axial strain measurements for rat-tail vertebrae loaded in axial compression, calculated by image registration.

\begin{tabular}{lcccccc}
\hline Rat \# & $\begin{array}{c}\text { Average } \\
\text { strain }\end{array}$ & $\begin{array}{c}\text { Strain in 10th } \\
\text { percentile }\end{array}$ & $\begin{array}{c}\text { Strain in 90th } \\
\text { percentile }\end{array}$ & $\begin{array}{c}\text { Median } \\
\text { strain }\end{array}$ & $\begin{array}{c}\text { Standard } \\
\text { deviation }\end{array}$ & $\begin{array}{c}\text { Load applied } \\
(\mathrm{N})\end{array}$ \\
\hline 1 & -0.0437 & 0.0141 & -0.1600 & -0.0124 & 0.0811 & 27 \\
2 & -0.0080 & 0.0074 & -0.0222 & -0.0068 & 0.0216 & 30 \\
3 & -0.0160 & 0.0137 & -0.0323 & -0.0026 & 0.054 & 30 \\
4 & -0.0043 & 0.0042 & -0.0139 & -0.0014 & 0.014 & 30 \\
5 & -0.0100 & 0.0023 & -0.0258 & -0.0039 & 0.027 & 30 \\
6 & -0.0089 & 0.0058 & -0.0228 & -0.0034 & 0.029 & 30 \\
7 & -0.0083 & 0.0962 & -0.1195 & -0.0102 & 0.097 & 20 \\
8 & -0.0282 & 0.0033 & -0.1003 & -0.0117 & 0.048 & 32 \\
Average & $-\mathbf{0 . 0 1 5 9}$ & $\mathbf{0 . 0 1 8 4}$ & $-\mathbf{0 . 0 6 2 1}$ & $-\mathbf{0 . 0 0 6 5}$ & $\mathbf{0 . 0 5 5 6}$ & $\mathbf{2 9}$ \\
\hline
\end{tabular}




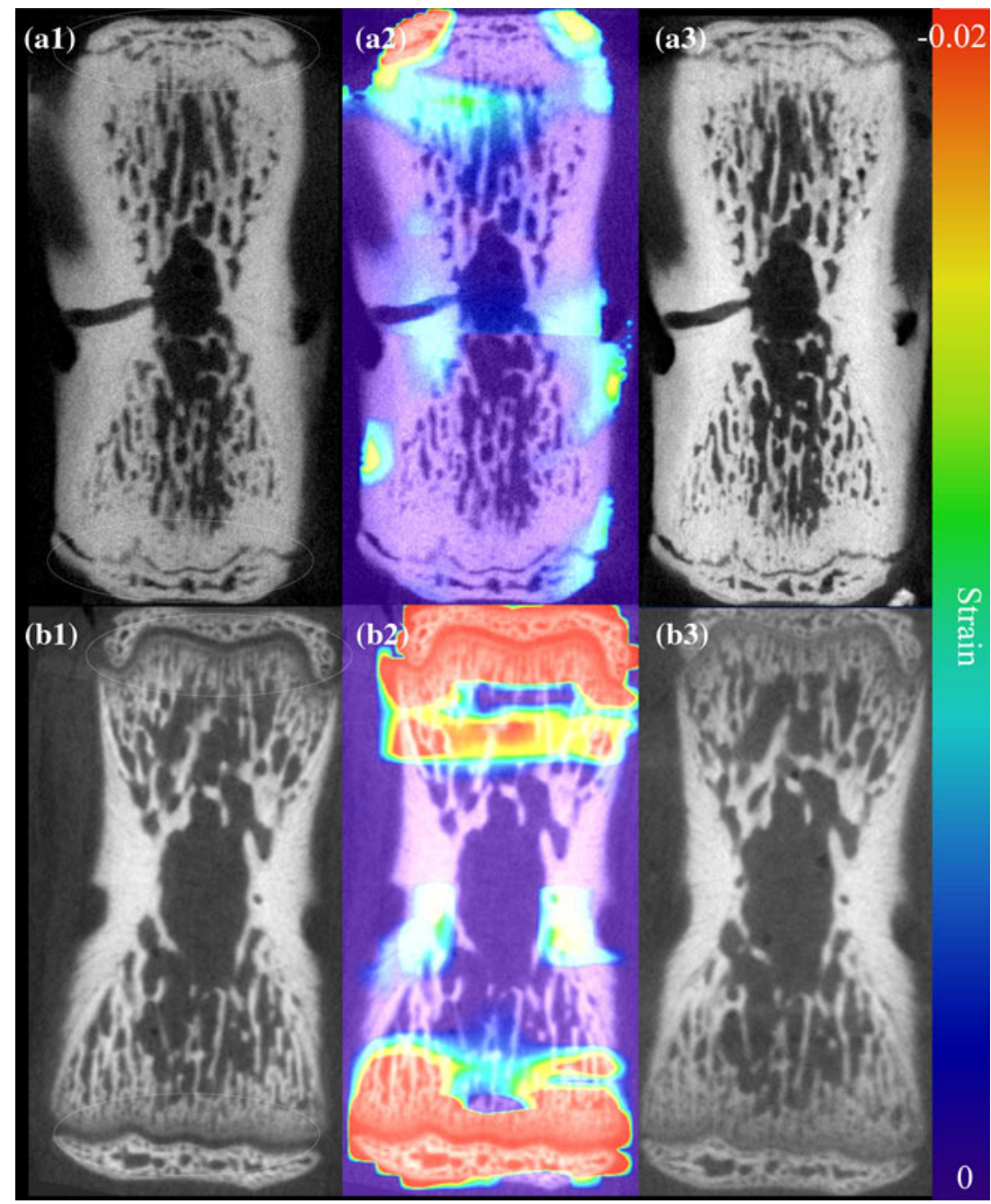

FIGURE 3. Coronal slices of rat vertebrae CT scans. A representative specimen from rigid growth plate group in the (a1) unloaded configuration with the growth plates encircled in ovals, (a2) with axial strain contours plotted on top of the unloaded configuration, and (a3) in the loaded configuration. (b1-3) The same configurations for a representative specimen from the compliant growth plate group, yielding greater displacements and strains in the growth plate regions.

TABLE 2. Strain distribution comparison calculated from image registrations between specimens groups based on growth plate compliance.

\begin{tabular}{lcrr}
\hline Mean + STD & Compliant growth plate, $n=3$ & Rigid growth plate, $n=5$ & Overall, $n=8$ \\
\hline Minimum strain (10th percentile) & $0.038+0.042$ & $0.007+0.004$ & $0.018+0.030$ \\
Average axial strain & $-0.027+0.015$ & $-0.0094+0.004$ & $-0.016+0.013$ \\
Maximum strain (90th percentile) & $-0.127+0.025$ & $-0.023+0.006$ & $-0.062+0.052$ \\
Load applied (N) & $26+5$ & $30+0$ & $29+4$ \\
\hline
\end{tabular}

compression $\left(0.67-1.29 \mathrm{MPa},{ }^{5} 0.57-1.41 \mathrm{MPa}^{8}\right)$ than tension (23-48.6 MPa, $\left.{ }^{4} 17.7-27 \mathrm{MPa}^{8}\right)$, both of which are much lower than the apparent elastic modulus of trabecular bone $\left(67-450 \mathrm{MPa}^{15}\right)$.

The large difference in elastic modulus between the growth plate and the trabecular bone is consistent with the results of this investigation, with much of the strain being taken up by the growth plate. The high strains within the growth plate may well be within the physiologic realm corresponding with findings that high-compressive loads can decrease the rate of lengthening, which are thought to occur because of a decrease of size of hypertrophic cells. ${ }^{29}$ Stresses up to $0.2 \mathrm{MPa}$ (corresponding to approximately 0.33 strain) in compression have been shown to decrease the rate of lengthening of bone. ${ }^{24}$ 


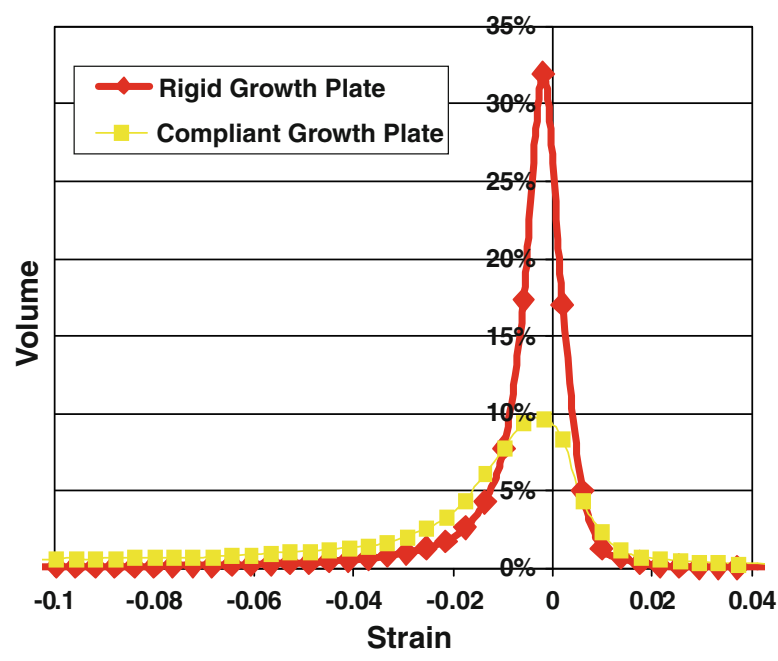

FIGURE 4. Comparison of the axial strain distributions of rigid and compliant growth plate groups. The compliant growth plate group has a much smaller peak.

302

303

304

305

306

307

308

309

310

311

312

313

314

315

316

317

318

319

320

321

322

323

324

325

326

327

328

329

330

331

332

333

334

335
Image registration yields a spatial strain distribution without having to make assumptions about the magnitude or distribution of applied loading or inherent material properties of the bony or soft tissues (as is required in finite element modeling). However, the method does assume that the structure that is being scanned remains intact and undergoes elastic deformation. The method will not properly measure strains in regions that have been damaged or undergone plastic-like deformations. The complexity and variability in material properties within the growth plate make it difficult to draw inferences about how $\mu \mathrm{CT}$-based measurements may predict its material behavior. Uncertainty in the material properties of the growth plate create a challenge in utilizing the finite element method to model the behavior of whole vertebrae and calculate similar spatial distributions of strain.

Bulk biomechanical testing is unable to determine the distribution of strain within specimens, and thus would not differentiate between trabecular and growth plate strains. The importance of the growth plate in the biomechanical response of rat vertebrae is more easily discernable through the application of image registration to calculate strain fields.

However, it should be noted that all specimens in the study contained growth plates and therefore definitive conclusions as to the effect of the presence of growth plates cannot be made. However, the more rigid growth plate group does provide a clue as to the behavior of vertebrae without growth plates, as the growth plate during maturation will fuse (in most species) and be remodeled to resemble the adjacent trabecular bone. Using image registration-based methods to investigate growth plate mechanics is limited by the ability of the technique to only capture the deformation between two distinct time points, therefore not allowing for the study of the possible path between the two points. This is particularly relevant in the growth plate as the viscoelastic properties ${ }^{21}$ are undoubtedly important to the function of the structure and they cannot be appreciated with the current method given $\mu \mathrm{CT}$ scanning times.

The mechanical role of the growth plate has previously been investigated by Sairyo et al. who modeled the pediatric spine using finite element analysis. In their work, the growth plates were modeled as significantly more compliant than the adjacent trabecular bone (10 MPa vs. $100 \mathrm{MPa}) .^{19}$ In agreement with our results, they showed that strains were more varied and greater with the presence of growth plates in whole pediatric vertebrae, compared to adult controls, with increased strains near the growth plate. ${ }^{19}$

Other work has further focused on strain patterns within the growth plate tissue. These investigations dealt with growth plates that were removed from bones prior to testing, thus did not include information on the surrounding bone. Villemure et al. investigating the mechanics of growth plates in vitro using confocal microscopy and texture correlation found large variability in strain distribution throughout the growth plate layers, with the greatest strains occurring in the reserve and hypertrophic zones. ${ }^{28}$ Villemure's results are consistent with the findings in this paper, in which growth plate strain distributions were also found to be highly variable within and between specimens [with large strains (up to 0.4) resulting from an applied 0.05 strain]. Similarly, Fujii et al. found that tensile failure predominantly occurred in the hypertrophic zone of the growth plate. ${ }^{8}$ Radhakrishnan et al. using atomic force microscopy also found differences in growth plate stiffness with depth, finding exponentially increasing stiffness from the reserve zone to the mineral zone. ${ }^{18}$

The maximum strains measured within this study (0.1-0.16) appear very high for bony structures prefailure. However, it is important to consider two important points when interpreting these high-strain values: first, they occur near and around the growth plate and second, the length scale on which they are measured lies between apparent and tissue level behavior. The apparent axial strains measured within this study (apparent level or average strains of 0.009 0.02 ) were similar to previously found apparent level strains in trabecular bone on the order of $0.01 .^{15}$ These large strains measured are in line with the strains that have been reported in the growth plate and for individual trabecular failure. Villemure et al. reported strains from 0.05 to 0.40 in the growth plate under 
391 compressive loading ${ }^{28}$ and Hernandez et al. measured

392 failure strains in individual trabeculae that varied from 3930.018 to $0.20 .^{11}$

394 The growth plate's extracellular matrix contains 395 proteoglycans, collagen, and water, and is modeled as 396 a biphasic material. ${ }^{5,8}$ The growth plate in long bones ${ }^{4}$ 397 and in vertebrae has an undulated structure (Fig. 1), 398 however regardless of the orientation of the specific 399 undulation of the growth plate, the cells within it grow 400 in lines that are aligned with the long axis of the 401 bone. $^{4,5}$ In our study, a similar undulated structure of 402 the growth plates within the vertebrae was found. 403 Interestingly, Cohen et al. found denser bone on the 404 proximal side, while in this study denser bone was 405 observed on the distal side of the growth plate. ${ }^{4}$ This 406 structural difference may have an effect on the per407 meability of the two sides, affecting the viscoelastic 408 response of the growth plate. ${ }^{5}$

409 Image registration was able to successfully quantify 410 the biomechanical response within the growth plate 411 due to the large contrast difference between the growth 412 plate and adjacent bone. However, the presence of the 413 growth plate limits the ability of the image registration 414 method to accurately spatially resolve strain in the 415 trabecular bone of whole rat vertebrae. Large strains in 416 the growth plate lead to adjacent bone appearing 417 highly strained due to the limited spatial resolution 418 possible with this image registration technique. The 419 growth plate hinders the image registration methods 420 from completely spatially resolving where the strain 421 occurs because there is no contrast within the structure 422 when it is imaged with CT. Further, the ability to 423 calculate accurate strain is dependent upon the ability 424 to accurately register subregions. These subregions 425 must have sufficient contrast texture to accurately find 426 the displacement. The growth plates have no internal 427 contrast on CT, thus the only features that are used to 428 accurately measure displacement in the region around 429 the growth plate is the surrounding trabecular bone 430 and the interface of the growth plate with the bone. 431 The limited spatial resolution possible with this tech432 nique due to the underlying structure indicates a 433 potential benefit of the utilization of other computa434 tional analysis approaches; whereas image registration 435 does not require as much a priori knowledge it never436 theless may result in lower resolution results than is 437 possible with the finite element method.

438 The ease of interpretation of the strain fields was 439 also limited by the loading technique. The load was 440 applied through the adjacent vertebral bodies and 441 intervertebral discs in order to represent a more 442 physiologic scenario. However, this resulted in load 443 application that was not entirely axial compressive, as 444 evidenced by the presence of limited tensile strains, 445 suggesting small amounts of bending. The presence of wide distribution of strains (Fig. 4) strongly suggests some asymmetry in the load applied; however, this may accurately represent the physiological loading scenario. This aberration of the loading is noted as a limitation of the loading protocol; however, load was predominantly applied in the axial direction.

\section{CONCLUSIONS}

Compressive loading of the rat-tail vertebrae resulted in strain concentrations within the growth plates. The amount of strain experienced by the growth plates was highly variable, yielding two distinct patterns demonstrating rigid and compliant growth plate behavior. The specimens with compliant growth plates showed greater strain concentrations within and around the growth plates with greater average whole specimen strain experienced than specimens possessing a more rigid growth plate. The observed strain concentrations within and adjacent to the growth plate may lead to increased fracture risk near the growth plate. This strain distribution may provide insight into the mechanical signals that cells experience during the formation of new bone with higher strains near the growth plate signaling cells to lay down more bone. Finally, the amount of strain within the growth plate is important to consider when interpreting mechanical testing data obtained from specimens that contain growth plates, which is commonly reported in the literature, ${ }^{14,16,20,22}$ as this study suggests strains are not uniformly distributed with high concentrations in and around the growth plate.

\section{ACKNOWLEDGMENTS}

This study was supported by the Canadian Institutes for Health Research and the Canadian Breast Cancer Foundation-Ontario Chapter. We also acknowledge the work of Tomas Skrinskas in assisting in the sample preparation.

\section{REFERENCES}

${ }^{1}$ Ballock, R. T., and R. J. O'keefe. The biology of the growth plate. J. Bone Jt. Surg. Am. 85A:715-726, 2003.

${ }^{2}$ Bay, B. K., T. S. Smith, D. P. Fyhrie, and M. Saad. Digital volume correlation: three-dimensional strain mapping using x-ray tomography. Exp. Mech. 39:217-226, 1999.

${ }^{3}$ Chiang, G. Design of a System to Study the Biomechanics of Metastatic Disease in Rat-Tail Vertebrae. Toronto: University of Toronto, 2003.

${ }^{4}$ Cohen, B., G. S. Chorney, D. P. Phillips, H. M. Dick, J. A. Buckwalter, A. Ratcliffe, and V. C. Mow. The 
microstructural tensile properties and biochemicalcomposition of the bovine distal femoral growth plate. J. Orthop. Res. 10:263-275, 1992.

${ }^{5}$ Cohen, B., G. S. Chorney, D. P. Phillips, H. M. Dick, and V. C. Mow. Compressive stress-relaxation behavior of bovine growth plate may be described by the nonlinear biphasic theory. J. Orthop. Res. 12:804-813, 1994.

${ }^{6}$ Eswaran, S. K., H. H. Bayraktar, M. F. Adams, A. Gupta, P. F. Hoffmann, D. C. Lee, P. Papadopoulos, and T. M. Keaveny. The micro-mechanics of cortical shell removal in the human vertebral body. Comput. Methods Appl. Mech. Eng. 196:3025-3032, 2007.

${ }^{7}$ Eswaran, S. K., A. Gupta, and T. M. Keaveny. Locations of bone tissue at high risk of initial failure during compressive loading of the human vertebral body. Bone 41:733-739, 2007.

${ }^{8}$ Fujii, T., S. Takai, Y. Arai, W. Kim, D. Amiel, and Y. Hirasawa. Microstructural properties of the distal growth plate of the rabbit radius and ulna: biomechanical, biochemical, and morphological studies. J. Orthop. Res. 18:87-93, 2000.

${ }^{9}$ Goss, P. E., S. Qi, R. G. Josse, K. P. Pritzker, M. Mendes, H. Hu, S. D. Waldman, and M. D. Grynpas. The steroidal aromatase inhibitor exemestane prevents bone loss in ovariectomized rats. Bone 34:384-392, 2004.

${ }^{10}$ Hardisty, M. R., and C. M. Whyne. Whole bone strain quantification by image registration: a validation study. J. Biomech. Eng. 131:064502, 2009.

${ }^{11}$ Hernandez, C. J., S. Y. Tang, B. M. Baumbach, P. B. Hwu, A. N. Sakkee, F. Van Der Ham, J. Degroot, R. A. Bank, and T. M. Keaveny. Trabecular microfracture and the influence of pyridinium and non-enzymatic glycationmediated collagen cross-links. Bone 37:825-832, 2005.

${ }^{12}$ Hoc, T., L. Henry, M. Verdier, D. Aubry, L. Sedel, and A. Meunier. Effect of microstructure on the mechanical properties of Haversian cortical bone. Bone 38:466-474, 2006.

${ }^{13}$ Horton, J. A., J. T. Bariteau, R. M. Loomis, J. A. Strauss, and T. A. Damron. Ontogeny of skeletal maturation in the juvenile rat. Anat. Rec. (Hoboken) 291:283-292, 2008.

${ }^{14}$ Ito, M., A. Nishida, A. Koga, S. Ikeda, A. Shiraishi, M. Uetani, K. Hayashi, and T. Nakamura. Contribution of trabecular and cortical components to the mechanical properties of bone and their regulating parameters. Bone 31:351-358, 2002.

${ }^{15}$ Keaveny, T. M., E. F. Morgan, G. L. Niebur, and O. C. Yeh. Biomechanics of trabecular bone. Ann. Rev. Biomed. Eng. 3:307-333, 2001.

${ }^{16}$ Lauing, K., R. Himes, M. Rachwalski, P. Strotman, and J. J. Callaci. Binge alcohol treatment of adolescent rats followed by alcohol abstinence is associated with sitespecific differences in bone loss and incomplete recovery of bone mass and strength. Alcohol 42:649-656, 2008.

${ }^{17}$ Perez-Quintian, F., A. Lutenberg, and M. A. Rebollo. Linear displacement measurement with a grating and speckle pattern illumination. Appl. Opt. 45:4821-4825, 2006.

${ }^{18}$ Radhakrishnan, P., N. T. Lewis, and J. J. Mao. Zonespecific micromechanical properties of the extracellular matrices of growth plate cartilage. Ann. Biomed. Eng. 32:284-291, 2004.

${ }^{19}$ Sairyo, K., V. K. Goel, A. Masuda, S. Vishnubhotla, A. Faizan, A. Biyani, N. Ebraheim, D. Yonekura, R. Murakami, and T. Terai. Three dimensional finite element analysis of the pediatric lumbar spine. Part II: biomechanical change as the initiating factor for pediatric isthmic spondylolisthesis at the growth plate. Eur. Spine J. 15:930935, 2006

${ }^{20}$ Sehmisch, S., M. Erren, T. Rack, M. Tezval, D. SeidlovaWuttke, J. Richter, W. Wuttke, K. M. Stuermer, and E. K. Stuermer. Short-term effects of parathyroid hormone on rat lumbar vertebrae. Spine (Phila Pa 1976), 34:2014-2021, 2009.

${ }^{21}$ Sergerie, K., M.-O. Lacoursière, M. Lévesque, and I. Villemure. Mechanical properties of the porcine growth plate and its three zones from unconfined compression tests. J. Biomech. 42:510-516, 2009.

${ }^{22}$ Shiraishi, A., S. Miyabe, T. Nakano, Y. Umakoshi, M. Ito, and M. Mihara. The combination therapy with alfacalcidol and risedronate improves the mechanical property in lumbar spine by affecting the material properties in an ovariectomized rat model of osteoporosis. BMC Musculoskelet. Disord. 10:66, 2009.

${ }^{23}$ Smith, T. S., B. K. Bay, and M. M. Rashid. Digital volume correlation including rotational degrees of freedom during minimization. Exp. Mech. 42:272-278, 2002.

${ }^{24}$ Stokes, I. A., D. D. Aronsson, A. N. Dimock, V. Cortright, and S. Beck. Endochondral growth in growth plates of three species at two anatomical locations modulated by mechanical compression and tension. J. Orthop. Res. 24:1327-1334, 2006.

${ }^{25}$ Sutton, M. A., A. K. Wong, and Y. J. Chao. Enhanceddisplacement measurement using a generalized formulation for double-aperture specklegrams. Exp. Mech. 23:348-353, 1983.

${ }^{26}$ Van Roy, P., E. Barbaix, M. De Maeseneer, C. Pouders, and J. Clarys. The anatomy of the neural arch of the lumbar spine, with references to spondylolysis, spondylolisthesis, and degenerative spondylolisthesis. In: Spondylolysis, Spondylolisthesis, and Degenerative Spondylolisthesis. Lippincott: Williams \& Wilkins, 2005, p 1.

${ }^{27}$ Veress, A. I., J. A. Weiss, G. T. Gullberg, D. G. Vince, and R. D. Rabbitt. Strain measurement in coronary arteries using intravascular ultrasound and deformable images. J. Biomech. Eng. 124:734-741, 2002.

${ }^{28}$ Villemure, I., L. Cloutier, J. R. Matyas, and N. A. Duncan. Non-uniform strain distribution within rat cartilaginous growth plate under uniaxial compression. J. Biomech. 40:149-156, 2007.

${ }^{29}$ Villemure, I., and I. A. F. Stokes. Growth plate mechanics and mechanobiology. A survey of present understanding. J. Biomech. 42:1793-1803, 2009.

${ }^{30}$ Zauel, R., Y. N. Yeni, B. K. Bay, X. N. Dong, and D. P. Fyhrie. Comparison of the linear finite element prediction of deformation and strain of human cancellous bone to 3D digital volume correlation measurements. J. Biomech. Eng. 128:1-6, 2006. 